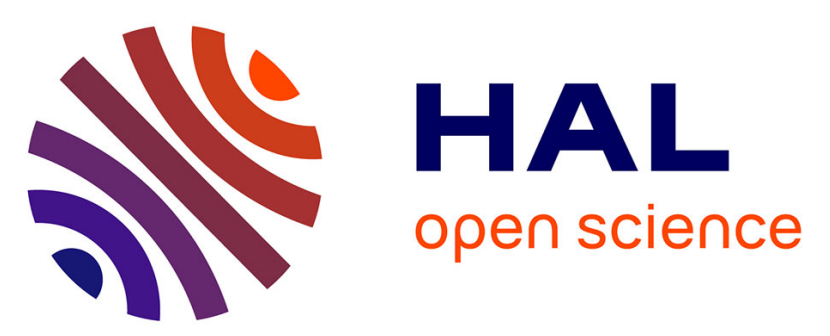

\title{
Gouvernance et innovation au sein des technopôles: le cas de Savoie Technolac
}

\author{
Rachel Bocquet, Sébastien Brion, Caroline Danièle Mothe
}

\section{To cite this version:}

Rachel Bocquet, Sébastien Brion, Caroline Danièle Mothe. Gouvernance et innovation au sein des technopôles: le cas de Savoie Technolac. Revue Française de Gestion, 2013, 39 (232), pp.101-118. 10.3166/RFG.232.101-118 . hal-00915161

\section{HAL Id: hal-00915161 \\ https://hal.science/hal-00915161}

Submitted on 6 Dec 2013

HAL is a multi-disciplinary open access archive for the deposit and dissemination of scientific research documents, whether they are published or not. The documents may come from teaching and research institutions in France or abroad, or from public or private research centers.
L'archive ouverte pluridisciplinaire $\mathbf{H A L}$, est destinée au dépôt et à la diffusion de documents scientifiques de niveau recherche, publiés ou non, émanant des établissements d'enseignement et de recherche français ou étrangers, des laboratoires publics ou privés. 


\section{Gouvernance \\ et innovation au sein des technopôles}

\section{Le cas de Savoie Technolac}

La question de savoir si la proximité organisationnelle peut être encouragée au sein des clusters, en se concentrant sur les KIBS (Knowledge Intensive Business Services), a été peu traitée dans la littérature. L'objectif de cet article est d'étudier le rôle relationnel que peut jouer la structure de gouvernance d'un cluster pour améliorer les performances d'innovation de ses membres KIBS à travers différentes pratiques. La recherche s'appuie sur un échantillon représentatif de 53 KIBS au sein de Savoie Technolac. Les résultats obtenus suggèrent que, outre les déterminants traditionnels de l'innovation des entreprises, la structure de gouvernance a un impact direct sur l'innovation des KIBS du technopôle et un rôle indirect de tiers pour les KIBS engagés dans des partenariats de $R \& D$. Cette recherche a des implications importantes pour l'évaluation des actions de la structure de gouvernance dans les technopôles. 
L 'objectif de cet article est d'étudier les performances d'innovation des membres d'un cluster particulier, à savoir un technopôle. Malgré l'importance de leurs activités de recherche, les technopôles français, souvent constitués de petites et moyennes entreprises (PME), voire de très petites entreprises (TPE), sont, dans la grande majorité des cas, caractérisés par de faibles relations interfirmes (Cooke, 2001), limitant ainsi leur potentiel d'innovation à un niveau individuel et collectif. Parmi les facteurs qui pourraient contribuer à une telle dynamique, le développement de formes non spatiales de proximité représente un facteur explicatif important (Rallet et Torre, 2007). À ce titre, Pecqueur et Zimmermann (2004) proposent de distinguer deux formes de proximité : l'une, qualifiée de proximité organisationnelle est basée sur des interactions directes entre les acteurs; 1'autre, la proximité institutionnelle n'implique aucune interaction directe mais repose sur des dispositifs intermédiaires de coordination. Cette distinction semble particulièrement pertinente pour introduire le rôle relationnel de la structure de gouvernance d'un cluster pour développer un environnement local propice à l'innovation des membres (Longhi et Quéré, 1991).

Les travaux sur les clusters se sont essentiellement concentrés sur l'impact des propriétés structurelles des clusters sur leur performance et leur évolution. Bocquet et Mothe (2009) ont montré que les caractéristiques des clusters affectent aussi leur mode de gouvernance. Ce résultat s'inscrit dans la lignée des études qui mettent en avant le rôle central des institutions dans la création de formes non spatiales de proximité (Pecqueur et Zimmermann, 2004; Rallet et Torre, 2007). L'école française de la proxi- mité (Torre, 2006) introduit explicitement la notion de «gouvernance territoriale » pour désigner le processus institutionnel et organisationnel permettant de coordonner des acteurs géographiquement proches. Il a été par ailleurs démontré que la «gouvernance territoriale », ou « locale », notamment dans le contexte français (Ehlinger et al., 2007 ; Bocquet et Mothe, 2009 ; Mendez et Bardet, 2009), joue un rôle clé dans la création de conditions favorables à l'innovation collaborative. Ceci pourrait être le cas dans les technopôles français, où les entreprises sont de petites tailles et n'ont pas nécessairement les ressources matérielles et cognitives pour percevoir les possibilités technologiques et/ou entrer dans des partenariats formels de R\&D. La structure de gouvernance du cluster pourrait ainsi jouer un rôle actif dans le développement de ces ressources ainsi que dans l'organisation d'interactions propices à l'innovation de ses membres.

Les recherches empiriques consacrées aux pratiques de gouvernance en matière d'innovation mises en œuvre par la gouvernance territoriale sont rares. Celles-ci sont vues essentiellement sous l'angle des acteurs clés de cette structure de gouvernance (Loubaresse, 2008, 2011; Ditter et Bobulescu, 2010) et ne permettent pas de mesurer l'effet direct de cette gouvernance sur la performance d'innovation des entreprises membres. L'originalité de cette recherche consiste donc à identifier les pratiques mises en œuvre par la structure de gouvernance du technopôle et à évaluer leur impact sur les performances d'innovation de ses membres. La première contribution de cet article est donc de combler un vide dans la littérature sur la question de savoir si la proximité organisationnelle 
peut être favorisée par l'intervention d'une structure intermédiaire telle que la structure de gouvernance du cluster, dimension qui n'a été jusqu'ici que très peu été prise en considération. La seconde contribution de cet article est de fournir une meilleure compréhension des déterminants de la performance de l'innovation des membres d'un technopôle. Nous mettons en exergue le rôle de la structure de gouvernance comme une source potentielle d'innovation - qui vient s'ajouter aux sources plus « traditionnelles » et déjà largement étudiées.

Outre l'intérêt théorique, la recherche présente aussi un intérêt empirique de par l'étude d'un échantillon représentatif d'entreprises spécifiques, à savoir les KIBS (Knowledge Intensive Business Services, entreprises à forte intensité en connaissances ou entreprises de prestation de service intellectuel ${ }^{1}$ ). La recherche empirique s'appuie sur un échantillon représentatif de 53 KIBS au sein de Savoie Technolac dans la région Rhône-Alpes. Les résultats mettent tout d'abord en évidence un effet direct de la structure de gouvernance sur l'innovation des entreprises grâce à deux types de pratiques: les services dédiés à la création d'entreprise et une activité de réseautage qui vise à mettre les entreprises en relation avec d'autres organisations. Les résultats montrent aussi un effet indirect de la structure de gouvernance sur l'innovation des KIBS, ces derniers ne pouvant bénéficier de leur coopération avec les universités et laboratoires de recherche sans la médiation de la structure de gouvernance. Ces résultats témoignent du rôle relationnel fort des structures de gouvernance, renforçant ainsi le bien-fondé de l'obligation faite par les politiques de mettre en place des structures de gouvernance au sein des clusters français.

\section{I - INNOVATION ET GOUVERNANCE DES TECHNOPÔLES}

Le phénomène technopolitain et les parcs technologiques associés (ou technopôles) ont connu une forte croissance dans de nombreux pays développés depuis la fin des années 1970. Malgré leur relative ancienneté, notamment par rapport à d'autres types de cluster (comme les pôles de compétitivité), les technopôles toutefois sont loin de présenter un modèle homogène de l'innovation territoriale. Cooke (2001) identifie d'ailleurs deux types de technopôles, qualifiés de linéaires et interactifs, avec des propriétés et des performances distinctes. À la différence des technopôles linéaires, les technopôles interactifs seraient dotés de dispositifs ou ressources de médiation non plus uniquement considérés au niveau individuel mais également comme des ressources de coordination collective. Une telle perspective semble particulièrement pertinente pour établir le rôle que peut jouer une structure de gouvernance quand elle doit faire face à un manque de proximité organisationnelle au sein du cluster. Elle agit comme un acteur central qui joue le rôle stratégique d'une « organisation leader » (Provan et Kenis, 2008), un centre de décision qui maintient le réseau, les actions collectives et décisions majeures pour le réseau étant prises en charge par cet acteur référent unique (Provan et Kenis, 2008).

La structure de gouvernance apparaît nécessaire pour créer une dynamique collec-

1. Nous remercions un évaluateur anonyme pour cette suggestion de traduction de la notion de KIBS. 
tive entre des acteurs hétérogènes, asymétriques, et souvent déficitaires en ressources et en capacités d'interaction comme le sont souvent les PME d'un technopôle. Cette idée est au cœur de la notion de « gouvernance territoriale » (Leloup et al., 2005) ou « locale » (Gilly et Wallet, 2001), héritée des travaux de "l'école de la proximité ». La gouvernance territoriale, en tant que structure de pilotage et d'animation, garantit un « processus dynamique de confrontation et d'ajustement entre des acteurs hétérogènes mais géographiquement proches » (ibid, p. 556). Elle apparaît comme un mode de gouvernance plus adapté que la gouvernance associative propre aux districts industriels compte tenu de la forte hétérogénéité des acteurs en présence : laboratoires de recherche, universités, instituts privés de formation, organisations professionnelles, collectivités locales, TPE/PME, grandes entreprises, etc. (Alberti, 2001; Bocquet et al., 2009). Une telle structure de gouvernance dédiée au pilotage (gouvernance stratégique) et à l'animation (gouvernance opérationnelle) permet de faire émerger des représentations et des valeurs partagées entre les acteurs et de mettre en œuvre des actions destinées à favoriser leurs interactions. Contrairement à la gouvernance associative, toutes ces actions sont réalisées par la structure de gouvernance elle-même, autour d'une équipe dédiée - et non par d'autres institutions. Ainsi, la gouvernance territoriale introduit une rupture avec la perspective égocentrée du réseau qui vise à comprendre le pilotage du réseau par l'un des membres (Josserand, 1997). Dans cette perspective, Bocquet et al. (2009) ont montré que les risques de captation des ressources du cluster par le pivot étaient très forts. La gouvernance territoriale conduit à privilégier une autre perspective du pilotage du réseau par une structure de gouvernance dédiée, une entité coordinatrice en charge de fixer un cadre propice à la coopération entre les acteurs du cluster issus d'horizons cognitifs variés.

Nous avançons que la gouvernance territoriale du cluster peut contribuer à favoriser la proximité organisationnelle qui fait défaut dans les technopôles linéaires - alors qu'elle est centrale dans les technopôles interactifs. La structure de gouvernance peut contribuer à améliorer la proximité cognitive quand celle-ci est faible en raison de la forte hétérogénéité interentreprise (en termes de taille, d'activité, de marchés, de statut, etc.) et/ou de favoriser une proximité relationnelle.

Lorsque la proximité géographique est observée sans aucune forme de proximité organisationnelle, les acteurs économiques ont peu de chance d'entretenir des relations directes (Torre, 2006). Ce constat semble particulièrement vrai lorsque le technopôle regroupe une forte proportion de petites entreprises (Bocquet et Mothe, 2009). Étudiant les clusters avec de fortes concentrations de PME, Bocquet et Mothe (2010) ont montré les difficultés qui se posent lorsqu'on tente d'établir des synergies entre les PME en raison de leur comportement individualiste et de leurs difficultés à percevoir les opportunités d'innovation. Bien que la coopération et l'utilisation de sources externes soient des dimensions essentielles à leur activité d'innovation, toutes les PME n'ont pas la capacité à absorber les ressources externes et/ou sont même réticentes à établir de telles relations. La fertilisation croisée étant l'une des principales fonctions d'un technopôle, créer des ressources de médiation (proximité institutionnelle) 
lorsque la proximité organisationnelle est faible (proximités à la fois cognitive et relationnelle) représente un véritable enjeu pour la structure de gouvernance dudit technopôle. Pour certains auteurs, l'ancrage territorial apparaît précisément lorsque le cluster (proximité géographique) est capable de générer des effets de proximité organisationnelle et institutionnelle sur la base de l'interaction et de la coopération entre les unités opérant dans la même zone géographique. Par ses actions, la structure de gouvernance peut aider à développer un environnement propice à l'innovation des membres.

L'émergence d'un technopôle interactif peut donc résulter de l'établissement d'une structure de gouvernance locale capable de développer à la fois une proximité cognitive de manière à créer un sentiment d'appartenance commune qui lie les acteurs et une proximité relationnelle pour favoriser l'émergence de collaborations en matière d'innovation. La structure de gouvernance de technopôles devrait jouer un rôle important dans la stimulation de l'innovation grâce à la qualité de l'ancrage territorial et de ses actions de mise en réseau.

\section{Ancrage territorial}

Au-delà des services et des infrastructures développées pour les entreprises d'accueil (ressources génériques), la structure de gouvernance du technopôle est amenée à générer une diversité et une complémentarité entre les activités des entreprises membres. La combinaison d'une stratégie commune, de l'implication des acteurs dans les réseaux sociaux, personnels ou professionnels, et de la proximité géographique permet en effet de générer des ressources et des services spécifiques aux entreprises du cluster. La structure de gouvernance, à tra- vers le développement d'actions collectives et individuelles, peut également encourager les occasions de rencontres, formelles ou informelles, entre les acteurs. Ceci peut être réalisé grâce à des réunions thématiques ou des séminaires et à l'échange d'informations entre les membres. La structure de gouvernance doit aussi assurer la cohérence et la continuité du marché du travail local. Les entreprises ont besoin d'un stock suffisant de main-d'œuvre techniquement qualifiée, en particulier de scientifiques et d'ingénieurs pour absorber de nouvelles technologies, les modifier, pour créer et transférer de nouvelles informations technologiques. La capacité à recruter du personnel technique qualifié peut permettre aux entreprises d'échanger des savoir-faire et de profiter des retombées d'apprentissage localisé (Longhi et Quéré, 1991).

\section{Réseau}

La structure de gouvernance peut également jouer un rôle majeur dans la mise en réseau des parties prenantes afin de développer des synergies. Cette dimension de réseau a un caractère formel ici, par opposition aux relations informelles qui caractérisent l'ancrage territorial. Les incubateurs technologiques contribuent également à la promotion de l'innovation. La structure de gouvernance peut soutenir le développement d'une base de connaissances commune entre les membres, considérée comme un dispositif de traduction essentiel entre les différentes communautés (Bocquet et Mothe, 2010). L'accès à de nouvelles compétences et ressources est un motif clé dans les partenariats de recherche. Les relations université-industrie sont considérées comme un déterminant de la performance de l'innovation, y compris 
pour les PME. Ainsi, Romijn et Albaladejo (2002) fournissent des preuves que les interactions avec les institutions scientifiques et les fournisseurs de services (y compris d'origine publique) permettent d'augmenter les performances d'innovation des petites entreprises. Mais ils montrent aussi que l'origine et l'histoire comptent, et que cet effet positif n'est acquis que lorsque les propriétaires ou les managers des PME proviennent de ces institutions. Cette idée est conforme à la littérature qui montre que la coopération n'est pas spontanée, soulignant ainsi l'importance des réseaux relationnels. Et, bien que les contacts personnels et les liens sociaux entre acteurs soient considérés comme nécessaires à l'innovation, certains considèrent qu'ils ne sont pas suffisants pour accéder à la connaissance, souvent incrustée dans des équipes communes de recherche scientifique et industrielle.

La structure de gouvernance du cluster peut jouer un rôle actif dans le soutien de l'innovation en facilitant les spin-offs, de dépôt de brevets ou de licences, canaux essentiels de transferts de technologie. Or, peu d'attention a été accordée à la façon dont les petites entreprises, qui disposent souvent d'aucune ou d'un niveau relativement faible de capacité d'absorption (Cohen et Levinthal, 1990), s'engagent dans la recherche de technologies et de connaissances. Les auteurs soulignent le rôle d'un type spécifique d'intermédiaire technologique, à savoir les centres techniques et de recherche, dans le renforcement de leur capacité d'absorption. Ils mettent en évidence les multiples activités mises en œuvre par cet intermédiaire et montrent comment les entreprises n'ayant pas de capacité d'absorption sont aidées par ce dernier. Des travaux antérieurs ont cherché à évaluer plus spécifiquement le rôle et les compétences des « animateurs » (Ditter et Bobulescu, 2010) ou des «pilotes" (Loubaresse, 2008, 2011) de différents types de clusters. Toutefois, en privilégiant une approche qualitative auprès de ces acteurs, les effets de leurs actions sur les performances individuelles des entreprises et sur la performance à l'échelle collective restent difficiles à appréhender. À la différence de ces travaux, nous proposons une évaluation plus directe des actions de la structure de gouvernance du point de vue des entreprises membres du cluster.

\section{II - ÉTUDE EMPIRIQUE DU TECHNOPÔLE SAVOIE TECHNOLAC}

Cette recherche s'appuie sur une démarche quantitative permettant d'appréhender les perceptions des membres d'un technopôle français. Le fait d'avoir privilégié le point de vue des entreprises a pour but d'aboutir à une mesure directe (bien qu'établie de manière déclarative) de l'impact des actions de la structure de gouvernance. Elle se différencie nettement de la grande majorité des travaux qui, face au manque de données, ont privilégié des mesures indirectes à travers notamment les perceptions des membres de la structure de gouvernance. Les actions mises en œuvre par la structure de gouvernance ont été identifiées sur la base de notre grille d'analyse théorique. Ainsi, nous avons distingué 1) les actions favorisant l'ancrage territorial des membres du cluster et 2) les actions favorisant la mise en réseau des membres. L'identification de l'ensemble des actions a été réalisée à partir de l'intranet de Savoie Technolac et validée 
par la directrice de l'innovation de ST, qui est à la fois membre de la gouvernance stratégique et opérationnelle du cluster.

Nos questions concernant l'innovation sont strictement identiques à celles des enquêtes CIS (Community Innovation Surveys) pour éviter tout biais potentiel relatif à leur formulation. Il a été montré que les réponses, basées sur du déclaratif, ne différaient pas de plus de $5 \%$ par rapport aux données objectives, notamment sur les questions relatives à l'innovation des entreprises (Mairesse et Mohnen, 2010). Ces enquêtes sont aujourd'hui largement utilisées par les chercheurs en innovation. Le recours à une démarche quantitative, et aux équations structurelles en particulier, pour recueillir les perceptions des membres de Savoie Technolac et mesurer leurs effets sur leur performance d'innovation, offre un double avantage, elle permet d'évaluer : 1) la pertinence empirique des mesures de la gouvernance issues de la littérature, et 2) si ces variables explicatives agissent sur la performance d'innovation.

L'étude du technopôle Savoie Technolac, réalisée en février-juin 2009, est basée sur une enquête auprès de 88 KIBS situés dans le technopôle. Des questionnaires en ligne ont été envoyés à tous les dirigeants des entreprises. Après deux relances, nous avons reçu 53 questionnaires valides, ce qui correspond à un taux de retour de $62 \%$. Ce taux de réponse exceptionnellement élevé est dû à l'appui institutionnel fourni par la directrice de l'innovation de Savoie Technolac ${ }^{2}$ qui a coordonné les campagnes de relances. L'échantillon final est représentatif de la population des entreprises en termes de taille et d'activités.

\section{Origine et histoire}

Savoie Technolac a été créé en 1987 après la fermeture d'une base aérienne militaire au Bourget-du-Lac. Suivant le modèle de la Silicon Valley, le technopôle a émergé d'une volonté commune de personnalités politiques et économiques de développer un nouveau territoire associant l'université, la recherche et les services de haute technologie. Savoie Technolac est actuellement composé de près de 180 entreprises, 19 laboratoires de recherche et 69 programmes scientifiques et techniques d'enseignement supérieur couvrant quatre secteurs d'activité principaux : 1) l'énergie solaire et les écotechnologies; 2) les technologies de l'information, de l'électronique et de la communication, 3) la conception, le développement et le prototypage d'équipements industriels, et 4) les matériaux plastiques et composites. Savoie Technolac, à quelques pas du lac du Bourget, a centré son développement autour des préoccupations environnementales. En 2005, cette dimension environnementale a été renforcée avec l'implantation de l'Institut national de l'énergie solaire (INES). Savoie Technolac accueille également des entreprises qui appartiennent au pôle de compétitivité Tenerrdis axé sur les énergies renouvelables. Cette conversion économique a fait de la «Solar Valley » une référence pour l'industrie solaire en France.

2. Nous avons pris la précaution de contrôler le biais potentiel de sélection en comparant les caractéristiques (en termes de taille et d'activité) des 53 KIBS composant notre échantillon à celles des KIBS non répondantes. Les tests de comparaison de moyennes effectués révèlent que les différences sont non significatives, attestant de la qualité de notre échantillon. 


\section{Description des données}

Savoie Technolac comprend une proportion importante de très petites entreprises (moins de 10 salariés). La plupart des entreprises opèrent dans des activités de services, en particulier dans les services aux entreprises à forte intensité de connaissances (KIBS) : conseil, ingénierie ou R\&D. Nous nous concentrons ici sur ces KIBS et sur leurs performances en termes d'innovation. Seule la moitié des 53 KIBS de notre échantillon est effectivement engagée dans l'innovation (58,5\% dans l'innovation produit, 45,3\% dans l'innovation service).

La gouvernance de Savoie Technolac est constituée d'un syndicat mixte de collectivités locales, représentant le département de la Savoie, Chambéry Métropole et la Communauté de Communes du Lac du Bourget. Cette structure de gouvernance publique traduit la volonté des institutions publiques locales de conserver leur indépendance vis-à-vis des politiques publiques régionales ou nationales. Depuis sa création, les institutions locales ont décidé de mettre en place une équipe permanente en charge du pilotage et de l'animation du technopôle. L'équipe de direction de Savoie Technolac comporte douze membres « qui travaillent en étroite collaboration avec un réseau de partenaires distincts pour servir les meilleurs intérêts du territoire $»^{3}$. Ces partenaires sont dotés d'expertises complémentaires tels que l'innovation et le transfert technologique (CRITT), le financement de l'innovation (ARDI, OSEO), la propriété industrielle et intellectuelle (INPI), l'internationalisation et le développement économique (chambres consulaires, les conseils départementaux et régionaux). À côté du directeur général en charge de la stratégie globale et du directeur administratif et financier, l'équipe remplit les deux missions d'ancrage territorial et de mise en réseau.

\section{Ancrage territorial}

S'appuyant sur trois ressources principales - large espace disponible dans un environnement très attractif, proximité de l'université et des installations universitaires, et sources de financement, Savoie Technolac a réussi à attirer les PME ainsi que les départements scientifiques de l'université de Savoie. Le technopôle aide les entreprises à s'installer et les start-up innovantes par le biais d'un incubateur. 50,1\% des entreprises actuelles ont été incubées, attestant du dynamisme de l'incubateur et du rôle de spin-off joué par le technopôle. Deux tiers des KIBS (69,8 \%) s'appuient sur le marché du travail local. Afin d'ancrer les entreprises sur le territoire, Savoie Technolac propose un large éventail de services aux entreprises tels que l'incubateur, le conseil personnalisé, l'aide pour la recherche de soutien financier, ou autres événements informels de mise en relation (petits déjeuners thématiques, conférences, journées portes ouvertes, etc.) pour diffuser l'information, faciliter les rencontres entre les entreprises et créer un ancrage territorial dynamique. Ces services sont utilisés par $41,5 \%$ des membres.

\section{Réseau}

Savoie Technolac développe une stratégie de mise en relation des entreprises avec des partenaires sur site et/ou hors site pour l'innovation. L'équipe de gouvernance a mis $24,5 \%$ des KIBS en relation avec un par-

3. Site web de Savoie Technolac, http://www.savoie-technolac.com/125-equipe.htm (consulté le 15 juillet 2012). 
tenaire potentiel d'innovation, 18,9\% avec des clients, et 13,2\% avec un fournisseur. Cette mise en réseau a été réalisée au niveau individuel. Ce ne sont pas des actions collectives mais des relations spécifiques entre deux acteurs qui sont initiées par la structure de gouvernance. Développer la coopération industrie-recherche grâce à des incitations (actions comme les rencontres, petits déjeuners, réunions avec experts, mise en relation, etc.) afin de développer les possibilités de transfert de technologie est une priorité pour la structure de gouvernance de Savoie Technolac.

Savoie Technolac a pris un certain nombre d'initiatives stratégiques visant à construire des réseaux d'innovation aux niveaux local et global. Membre d'un réseau d'innovation dédié aux technopôles et incubateurs français, il est également impliqué dans deux pôles de compétitivité en Rhône-Alpes: Tenerrdis, dédié aux énergies renouvelables, et Plastipolis, spécialisé dans les matières plastiques et composites. Il a également développé des liens avec d'autres parcs scientifiques au niveau international, tels le Metropolitan Technopark (Québec) et Techno Park (Montréal). Toutefois, comme le montrent les statistiques descriptives, les collaborations en matière d'innovation doivent encore être renforcées.

\section{III - MÉTHODES ET RÉSULTATS}

Nous avons utilisé la modélisation par équations structurelles PLS (Partial Least Square) pour traiter les données issues de l'enquête (version Smart PLS 2.04). La méthode PLS est pertinente quand il s'agit d'évaluer des relations prédictives entre variables et pour des analyses visant à la construction d'une théorie (Wold, 1985). PLS est bien adapté à la nature exploratoire de notre approche compte tenu des recherches limitées sur la gouvernance et les déterminants de l'innovation dans les technopôles. L'analyse à l'aide de PLS se fait en deux étapes :

1) la première vise à valider empiriquement la pertinence des différentes mesures issues des dimensions identifiées dans la littérature théorique (cf. tableau 1: les dimensions sont toutes issues de la littérature);

2) la seconde cherche à évaluer les dimensions explicatives du modèle structurel. Pour mettre en évidence l'impact de la structure de gouvernance du technopôle sur l'innovation, nous avons construit deux modèles successifs, qui correspondent à deux modes d'intervention (direct et indirect) de la structure de gouvernance.

Le premier modèle (PLS1) considère la structure de gouvernance comme un (simple) prestataire de services. Il capte l'impact direct de la structure de gouvernance sur l'innovation des KIBS à travers la fourniture de différents services. Le deuxième modèle (PLS2) est hérité de la littérature sur la gouvernance des clusters et conçoit la structure de gouvernance comme un intermédiaire capable de soutenir un partenariat. Il met en évidence l'effet modérateur de la structure de gouvernance sur la performance des réseaux d'innovation. 
Tableau 1 - Description des variables

\begin{tabular}{|c|c|c|}
\hline & Items & Mesures \\
\hline \multicolumn{3}{|l|}{ Variables indépendantes } \\
\hline $\begin{array}{l}\text { Ancrage territorial } \\
\text { (ServiceCrea) } \\
\text { (Cooke et al., 2007; } \\
\text { Grossetti, 2000; } \\
\text { Carluer, 2006) }\end{array}$ & $\begin{array}{l}\text { Durant ces } 3 \text { dernières années, votre entreprise a-t-elle eu } \\
\text { recours aux services offerts par Savoie Technolac? } \\
\text { Services dédiés à la création d'entreprise (incubateurs, } \\
\text { conseils personnalisés, aides à la recherche de fonds, etc.) }\end{array}$ & Binaire \\
\hline $\begin{array}{l}\text { Activité de mise } \\
\text { en relation de } \\
\text { la gouvernance } \\
\text { (NetworkingST) } \\
\text { (de Jong et Marsili, 2006; } \\
\text { Favoreu et al., 2008) }\end{array}$ & $\begin{array}{l}\text { Durant ces } 3 \text { dernières années, l'équipe de Savoie } \\
\text { Technolac vous a-t-elle mis en relation avec une autre } \\
\text { organisation localisée sur le site ou pas: } \\
\text { 1) Un partenaire pour innover (RelaPart) } \\
\text { 2) Un client (RelaClst) } \\
\text { 3) Un fournisseur (RelaFost) }\end{array}$ & $\begin{array}{l}\text { Binaire } \\
\text { Binaire } \\
\text { Binaire }\end{array}$ \\
\hline $\begin{array}{l}\text { Recours au marché } \\
\text { du travail local } \\
\text { (EmploiST) } \\
\text { (Longhi et Quéré, 1991) }\end{array}$ & $\begin{array}{l}\text { Durant ces } 3 \text { dernières années, votre entreprise a-t-elle } \\
\text { utilisé le marché du travail local ? } \\
=1 \text { si l'entreprise a eu recours au marché du travail }\end{array}$ & Binaire \\
\hline $\begin{array}{l}\text { Coopération } \\
\text { de recherché comme } \\
\text { source d'innovation } \\
\text { (CollUniST) } \\
\text { (Romijn et Albaladejo, } \\
\text { 2002) }\end{array}$ & $\begin{array}{l}\text { Durant ces } 3 \text { dernières années, votre entreprise a-t-elle } \\
\text { eu recours pour sa recherche aux sources d'innovation } \\
\text { suivantes: } \\
=1 \text { si l'entreprise a collaboré avec des universités ou } \\
\text { instituts de recherche localisés sur le technopôle. }\end{array}$ & Binaire \\
\hline $\begin{array}{c}\begin{array}{c}\text { Autres sources } \\
\text { d'innovation } \\
\text { (SourcesInno) }\end{array} \\
\text { (de Jong et Marsili, 2006; } \\
\text { Favoreu } \text { et al., 2008) }\end{array}$ & $\begin{array}{l}\text { Durant ces } 3 \text { dernières années, votre entreprise a-t-elle eu } \\
\text { recours aux sources d'innovation suivantes: } \\
\text { 1) Collaboration avec les clients (SourcClSrv) } \\
\text { 2) Collaboration avec les fournisseurs (SourcForSrv) } \\
\text { 3) Collaboration avec les concurrents (SourcConcSrv) }\end{array}$ & $\begin{array}{l}\text { Binaire } \\
\text { Binaire } \\
\text { Binaire }\end{array}$ \\
\hline \multicolumn{3}{|l|}{ Variable dépendante } \\
\hline $\begin{array}{l}\text { Innovation de produits } \\
\text { ou de services } \\
\text { (InnoPdtSrv) } \\
\text { (CIS, 2008) }\end{array}$ & $\begin{array}{l}\text { Durant ces } 3 \text { dernières années, votre entreprise a-t-elle } \\
\text { introduit sur le marché: } \\
\text { 1) Des nouveaux produits (InnoPdt) } \\
\text { 2) Des nouveaux services (InnoSrv) }\end{array}$ & $\begin{array}{l}\text { Binaire } \\
\text { Binaire }\end{array}$ \\
\hline
\end{tabular}

\section{Validation empirique des variables}

Dans la méthode des équations structurelles, la validation empirique des variables composées de plusieurs items repose sur plusieurs indicateurs. Une première série d'indicateurs (variance moyenne extraite, AVE, et fiabilité composite) permet de vérifier la convergence des items avec la 
variable qu'ils sont censés représenter. Le tableau 2 montre que, pour chaque variable concernée, la valeur obtenue dans nos modèles dépasse le seuil requis pour ces deux indicateurs (respectivement 0,5 et 0,7 ). Une seconde série d'indicateurs permet de s'assurer que les items ne sont pas liés à d'autres variables que celles auxquelles ils sont destinés. Nous constatons que chaque item obtient un poids factoriel supérieur sur l'axe factoriel représentant leur variable de référence, comparativement au poids factoriel obtenu sur les autres axes qui sont moins élevés ${ }^{5}$. Un indicateur complémentaire permet de garantir que les racines carrées des AVE de chaque variable composée sont supérieures aux corrélations des autres variables du modèle. Notre modèle satisfait également cette dernière condition ${ }^{6}$.

\section{Résultats et discussion}

Les résultats des deux modèles PLS1 et PLS2 sont présentés dans le tableau 3. Ces résultats montrent qu'au-delà des déterminants classiques de la performance d'innovation des entreprises, la structure de gouvernance joue un rôle significatif. Compte tenu des niveaux relativement élevés des valeurs de coefficients de corrélation $\left(\mathrm{R}^{2}\right)$ dans les deux modèles, on peut en déduire que les variables choisies pour mesurer les pratiques de gouvernance de l'innovation ont un impact déterminant sur la performance d'innovation des entreprises. $\mathrm{La}$ légère augmentation de ces coefficients dans le second modèle nous conduit à le préférer au premier modèle.

Nos résultats mettent en évidence des effets directs et indirects de la structure de gouvernance des clusters sur la performance d'innovation des KIBS.

Notre premier modèle met en lumière l'impact de la qualité de l'ancrage territorial sur l'innovation produit/service. En effet, les deux variables EmploiST pour le marché local du travail $(\beta=0,334, \mathrm{t}=2,565$,

Tableau 2 - Fiabilité des mesures

\begin{tabular}{|c|c|c|c|c|}
\cline { 2 - 5 } \multicolumn{1}{c|}{} & \multicolumn{2}{c|}{ Modèle PLS1 } & \multicolumn{2}{c|}{ Modèle PLS2 } \\
\cline { 2 - 5 } & AVE & $\begin{array}{c}\text { Fiabilité } \\
\text { composite }\end{array}$ & AVE & $\begin{array}{c}\text { Fiabilité } \\
\text { composite }\end{array}$ \\
\hline NetworkingST & 0,615 & 0,823 & 0,615 & 0,823 \\
\hline SourcesInno & 0,603 & 0,752 & 0,603 & 0,752 \\
\hline InnoPdtSrv & 0,521 & 0,726 & 0,521 & 0,726 \\
\hline CollUniST * Networking & & & 0,750 & 0,900 \\
\hline
\end{tabular}

5. À l'exception de l'item SourcConSrv mesurant la collaboration avec les concurrents, cet item n'ayant pas atteint la valeur minimale de 0,5 sur l'axe factoriel SourcesInno. En accord avec les études antérieures sur les KIBS, les concurrents ne sont pas considérés comme des déterminants clés de l'innovation.

6. Le détail de ces tests est disponible auprès des auteurs. 
Tableau 3 - Résultats des deux modèles PLS

\begin{tabular}{|c|c|c|c|c|}
\hline & \multicolumn{2}{|c|}{ Modèle PLS1 } & \multicolumn{2}{|c|}{ Modèle PLS2 } \\
\hline Variable dépendante & \multicolumn{2}{|c|}{ InnovPdtSrv } & \multicolumn{2}{|c|}{ InnovPdtSrv } \\
\hline & $\beta$ & $t$ & $\beta$ & $t$ \\
\hline \multicolumn{5}{|l|}{ Variables indépendantes } \\
\hline CollUniST & $-0,195$ & $1,979 *$ & $-0,286$ & $2,267 *$ \\
\hline EmploiST & $\mathbf{0 , 3 3 4}$ & $2,565 * *$ & $\mathbf{0 , 3 3 9}$ & $2,614 * *$ \\
\hline NetworkingST & 0,322 & $2,940 * *$ & 0,242 & $1,892 *$ \\
\hline ServiceCrea & 0,262 & $2,593 * *$ & 0,240 & $2,233 *$ \\
\hline SourcesInno & 0,147 & 1,255 & 0,178 & $1,671^{*}$ \\
\hline $\begin{array}{l}\text { Effet modérateur } \\
\text { CollUniST x NetworkingST }\end{array}$ & & & 0,190 & $1,657 *$ \\
\hline $\begin{array}{r}\mathbf{R}^{2} \\
\triangle \mathbf{R}^{2}\end{array}$ & \multicolumn{2}{|c|}{0,367} & \multicolumn{2}{|c|}{$\begin{array}{c}\mathbf{0 , 3 8 6} \\
+\mathbf{0 , 0 1 9}\end{array}$} \\
\hline
\end{tabular}

$* \mathrm{p}<0,05$ (Test unilatéral : 1,645, df =499); ** $\mathrm{p}<0,01 ;(2,326, \mathrm{df}=499)$.

Figure 1 - Les effets directs de la structure de gouvernance sur la performance d'innovation (PLS1)

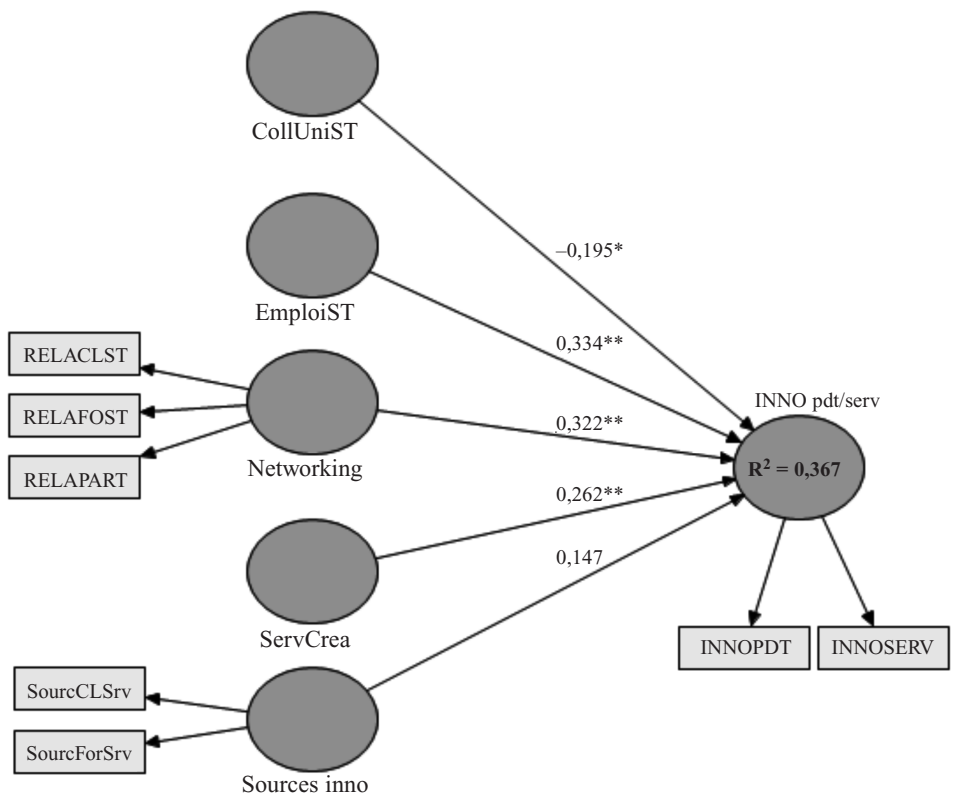

Note : les valeurs sur les flèches représentent le poids de la relation (ß).

Le niveau de significativité de la relation est noté : * $=p<0,05$ et $* *=p<0,01$. 
$\mathrm{p}<0,01$ ) et ServiceCrea pour les services spécifiques offerts par la structure de gouvernance $(\beta=0,262, \mathrm{t}=2,593, \mathrm{p}<0,01)$ ont un effet positif et significatif. Ce résultat est en accord avec les recherches précédentes sur l'innovation dans les KIBS. Il confirme que certains types d'environnements, des modes informels de transmission des connaissances et un marché du travail local dynamiques sont des déterminants majeurs de l'innovation des KIBS. À travers un marché du travail local très dynamique et des services dédiés au développement d'activité, les conditions de l'ancrage territorial sont supportées par la structure de gouvernance du technopôle et favorise ainsi l'innovation des entreprises. Pour les KIBS, un accès facilité au marché local du travail, suggère qu'elles peuvent accéder à des employés qualifiés, dotés d'un bon niveau scientifique et technique. Ce vecteur facilite le transfert et à la diffusion localisée des connaissances locales (Longhi et Quéré, 1991) et contribue à augmenter les capacités d'absorption des entreprises. Les services spécifiques au développement proposés par la structure de gouvernance du technopôle (aide au démarrage de start-up, déjeunés thématiques, conférences, club d'entrepreneurs, séminaires, etc.) permettent à des acteurs différents de se rencontrer et de se créer un langage commun. Ces services spécifiques apparaissent comme un prérequis à la création d'une proximité organisationnelle. Nos résultats suggèrent que la structure de gouvernance de Savoie Technolac exerce un rôle actif de médiation selon plusieurs schémas incitatifs qui in fine, favorise la performance d'innovation des KIBS.

En second lieu, nos résultats confirment l'impact positif et significatif des pratiques de mise en relation mises en ouvre par la structure de gouvernance sur l'innovation des entreprises $(\beta=0,322, \mathrm{t}=2,940$, $\mathrm{p}<0,01$ ), ces relations étant localisées autant à l'intérieur qu'à l'extérieur du technopôle. Alors que la littérature sur les KIBS a largement souligné l'influence des clients, fournisseurs et partenaires comme sources clés de l'innovation, aucune étude n'a encore montré le rôle clé de la structure de gouvernance dans cette mise en relation. Ce résultat fait écho aux travaux récents sur la gouvernance des clusters qui souligne le rôle médiateur de la structure de gouvernance, notamment pour les petites entreprises ne disposant pas des ressources matérielles et cognitives pour percevoir les opportunités technologiques et d'affaires. En accord avec les recherches précédentes (e.g. Bocquet et Mothe, 2010), la présence de nombreuses relations avec différents types d'acteurs est une dimension essentielle du processus d'innovation.

En troisième lieu, nos résultats ne permettent pas de confirmer l'effet positif attendu des sources d'innovation - indépendamment de la gouvernance. Bien qu'il n'y ait pas d'impact significatif des sources d'innovations issues des collaborations avec les clients et les fournisseurs, les résultats montrent un effet négatif significatif de CollUniSt, qui mesure les collaborations entre les entreprises et les universités/instituts de recherche du technopôle, sur l'innovation $(B=-0,195 \mathrm{t}=1,979, \mathrm{p}<0,05)$. Ce résultat suggère que certaines KIBS ne disposent pas de capacités d'absorption suffisantes pour tirer parti de leur partenariat de $R \& D$. D'une certaine manière, ceci est en opposition avec l'approche conventionnelle des KIBS qui souligne que ces entreprises ont précisément des capacités cognitives supérieures aux entreprises des 
autres secteurs. Toutefois, certaines caractéristiques des KIBS de notre échantillon (grande hétérogénéité sectorielle, entreprises de très petites tailles) peut expliquer cette différence ${ }^{7}$.

Dans le second modèle PLS (PLS2), nous avons voulu évaluer l'effet modérateur de la structure de gouvernance sur la relation entre les collaborations de recherche avec les universités/instituts de recherche et la performance de l'innovation. Nos résultats montrent un effet intéressant. En effet, alors que tous les effets identifiés dans PLS1 sont confirmés ici, les résultats indiquent clairement que les transferts de technologies issues de la collaboration avec les instituts de recherche existent bel et bien au sein du technopôle. Lorsque ce type de collaboration de recherche est combiné aux pratiques de mise en réseau de la structure de gouvernance (NetworkingSTxCollUniST), cette collaboration devient positive et significative $(\beta=0,190$, $\mathrm{t}=1,657, \mathrm{p}<0,05)$. Ce résultat signifie que lorsque des collaborations de recherches ont soutenues par la structure de gouvernance, l'impact de ces collaborations sur l'innovation est positif alors qu'il reste négatif lorsque ces collaborationnismes sont pas intermédiées par la structure gou-

Figure 2 - Les effets indirects (modérateur) de la structure de gouvernance sur la performance d'innovation (PLS2)

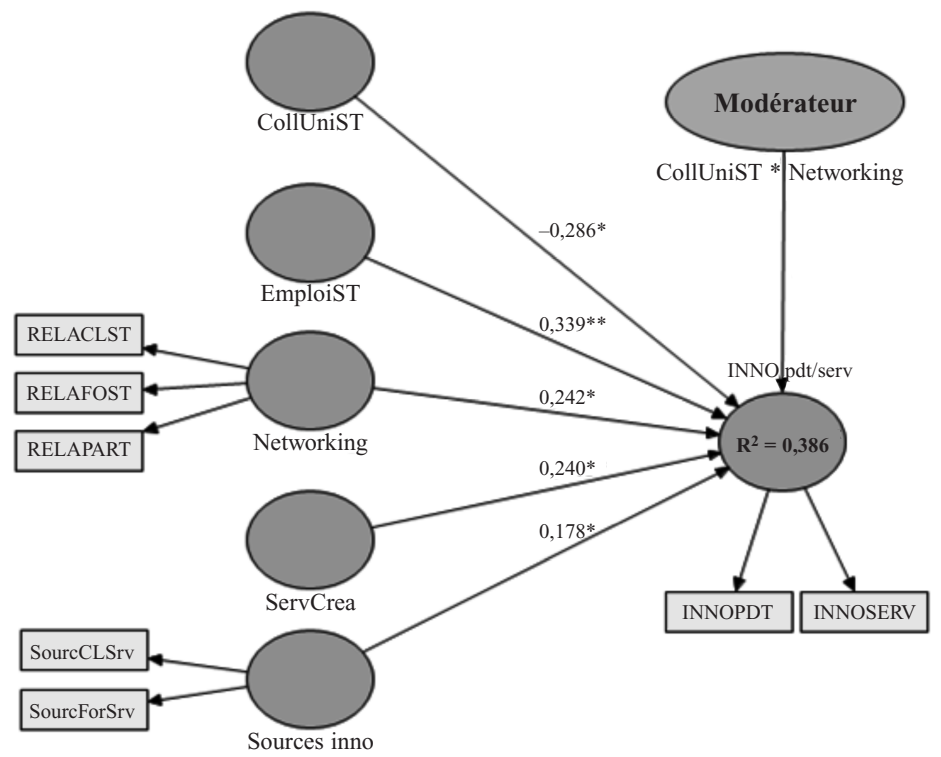

Note : les valeurs sur les flèches représentent le poids de la relation (ß).

Le niveau de significativité de la relation est noté : $*=p<0,05$ et $* *=p<0,01$.

7. Malheureusement, compte tenu de la petite taille de notre échantillon, nous ne sommes pas en mesure de confirmer l'influence de la taille et de l'activité des entreprises. 
vernance du technopôle. Ceci confirme que la structure de gouvernance peut jouer un rôle vital de «tierce partie » (Provan et Kenis, 2010) pour les entreprises disposant de ressources limitées pour poursuivre leurs efforts de R\&D, dans la lignée des résultats de Spithoven et al. (2011). Par ailleurs, ce deuxième modèle indique que les collaborations avec les acteurs amont et aval des entreprises (clients et fournisseurs) au cours de leur processus d'innovation stimule l'innovation (SourcesInno : $\beta=0,178, t=1,671, p<0,05)$. Ainsi, l'introduction de l'effet modérateur des pratiques mises en réseau de la structure de gouvernance augmente également l'impact de ces deux types de sources d'innovation, qui devient ici plus important. En somme, les réseaux renforcent l'effet de tous les types de sources d'innovation et de collaborations.

En définitive, l'un des résultats le plus remarquable est le rôle modérateur sur l'innovation de la mise en réseau par la structure de gouvernance sur les collaborations de recherche, non seulement ce lien reste significatif mais il change de signe et devient positif. La structure de gouvernance rend possible ce type de collaboration, qui sans elle, produit des effets néfastes sur l'innovation. Elle agit ainsi comme un intermédiaire mettant en relation les petites entreprises avec les institutions de recherche, offrant un support technique, financier et en termes de droits de la propriété industrielle, facilitant la recherche de partenaires et les collaborations internationales, etc. Ici, la structure de gouvernance ne se limite pas à un rôle passif de surveillance, mais met à disposition des entreprises des ressources stratégiques supplémentaires permettant de faire aboutir les projets de R\&D. La struc- ture de gouvernance du technopôle dispose ainsi d'une vision globale et stratégique des KIBS de son territoire, notamment à travers ces projets de recherche, ce qui permet de trouver rapidement le bon partenaire pour compléter des besoins en connaissance pour innover.

D'autres travaux empiriques ont pu mettre en évidence des effets similaires. Par exemple, Romijn et Albaladejo (2002) ont montré que les interactions avec des institutions scientifiques ont un effet positif sur l'innovation des petites entreprises uniquement lorsque le dirigeant est issu de ces institutions, ce dernier ayant ainsi développé des relations avec ces laboratoires. Ceci suggère que lorsque les petites entreprises (ici les KIBS) ne disposent pas d'expériences ou de relations d'antériorité avec les institutions de recherche, elles peuvent perdre beaucoup d'énergie, de ressources et de temps pour être en mesure de tirer parti de ces collaborations. Si l'on considère que la plupart des petites entreprises ne disposent pas de capacités d'absorption suffisantes (comme l'avaient déjà souligné Spithoven et al., 2011), le rôle d'intermédiaire technologique joué par la structure de gouvernance apparaît comme une ressource vitale des projets de collaboration de R\&D. Elle contribue à augmenter la capacité d'absorption en incitant à la mise en réseau de certains acteurs qui fournissent des ressources essentielles aux projets de R\&D.

\section{CONCLUSION}

Cet article est le premier à évaluer, à notre connaissance, l'effet des pratiques de la structure de gouvernance d'un cluster, en matière d'ancrage territorial et de mise en réseau, sur la performance d'innovation 
de ces entreprises membres. Cette analyse quantitative du rôle de la structure gouvernance dans un technopôle confirme non seulement le fait que les déterminants « traditionnels » influencent significativement la performance de l'innovation des entreprises co-localisées, mais souligne également le rôle joué par la gouvernance dudit technopôle. L'ancrage local et l'activité de réseau ont un impact positif significatif sur l'innovation produit des KIBS. Nos résultats sont donc doubles : d'une part, les KIBS ont la capacité d'absorber les connaissances par eux-mêmes (effet direct des pratiques d'ancrage et de réseau locaux); d'autre part, les entreprises de notre échantillon sont probablement trop petites pour bénéficier de collaborations en R\&D sans l'intermédiation de la gouvernance du technopôle (effet indirect). La capacité d'absorption des KIBS se situe donc à un niveau moyen de par l'effet combiné de la capacité d'absorption « naturellement » élevée des KIBS et de leur très petite taille. Plus généralement, ces deux effets de la gouvernance (direct et indirect) indiquent que les entreprises peuvent bénéficier des politiques de clusters (françaises) sur la base de stratégies volontaires et actives de création de connaissances.

Après vingt ans d'existence, Savoie Technolac s'appuie sur un marché du travail local dynamique et a réussi à inciter les entreprises à davantage collaborer et à entrer en réseau. Les entreprises et le technopôle sont toutefois encore dans une phase d'émergence caractérisée par un cluster de type « linéaire », et les collaborations entre recherche et industrie ne stimulent l'innovation produit des entreprises que lorsque ces dernières sont induites (et, du coup, suivies) par la gouvernance.
Ce fait n'est pas singulier car la plupart des études montrent qu'un certain laps de temps est nécessaire afin d'établir un réseau efficace de relations entre industrie, recherche et éducation, et une histoire commune entre les acteurs (Torre, 2006). Loubaresse (2011) avait également indiqué que le cycle de vie du réseau territorialisé était déterminant pour comprendre l'influence des compétences des pilotes opérationnels sur le pilotage des systèmes productifs locaux. Des exemples de succès de technopôles suggèrent une période de maturation de 15 à 20 ans (Lévesque et al., 1998) et un long processus de départ initial qui permet le développement d'un cadre suffisamment stable de relations interorganisationnelles.

Cette recherche n'est pas sans limites, en particulier en raison des spécificités de notre échantillon. Même s'il est représentatif de Savoie Technolac, il présente l'inconvénient d'être relativement petit et soumis à une grande hétérogénéité entre les sous-secteurs de KIBS - qui est un problème général pour toutes les recherches sur les KIBS. Les mesures utilisées pour évaluer le rôle et l'action de la gouvernance pourraient aussi être enrichies. En outre, la validité de nos résultats se limite au seul technopôle étudié. Des recherches futures pourraient être entreprises afin d'enrichir les connaissances empiriques relatives à la proximité organisationnelle et au rôle des pratiques de gouvernance visant à l'amélioration des performances d'innovation des entreprises. Plus précisément, une telle recherche pourrait bénéficier d'une étude qualitative approfondie sur le choix des «meilleures» pratiques de gouvernance en fonction du stade de développement du cluster, ou à sa maturité et à son position- 
nement par rapport à l'innovation technologique - plutôt produit ou service. Par ailleurs, le résultat sur le rôle modérateur de la gouvernance dans les relations de coopération avec les universités pourrait donner lieu à une étude qualitative approfondie afin de mieux comprendre ce rôle.

Cette recherche porte sur des entreprises de services particulières (les KIBS), traditionnellement plus prédisposées que les entreprises manufacturières de production à fonctionner en réseau et à innover, et avec des capacités d'absorption supérieures. Il était donc particulièrement intéressant d'étudier ces entreprises et l'impact que peut avoir la structure de gouvernance sur leur innovation puisqu'on peut a priori penser que cet impact serait renforcé pour l'industrie manufacturière. Les résultats pourraient donc être transférés à l'industrie manufacturière mais aussi à d'autres types de clusters, notamment aux pôles de compétitivité. Notre recherche tendrait ainsi à réhabiliter la politique des pôles de compétitivité français qui a imposé de telles structures de gouvernance en montrant le rôle clé que celles-ci peuvent jouer dans le renforcement de la capacité d'innovation des entreprises membres. L'évaluation des pôles pourrait comporter un volet sur l'évaluation des actions mises en place par ces structures de gouvernance.

\section{Bibliographie}

Amin A. et Cohendet P. (2005). "Geographics of knowledge formation in firms", Industry and Innovation, vol. 12, $\mathrm{n}^{\circ} 4$, p. 465-486.

Bocquet R. et Mothe C. (2009). «Gouvernance et performance des pôles de PME », Revue française de gestion, vol. 35, $\mathrm{n}^{\circ} 190, \mathrm{p} .101-122$.

Bocquet R. et Mothe C. (2010). « Le rôle des institutions publiques dans la gouvernance des pôles de compétitivité de PME », Revue Canadienne de Sciences Régionales, vol. XXXII, $\mathrm{n}^{\circ} 3$, p. 411-426.

Cohen W.M. et Levinthal D.A. (1990). "Absorptive capacity: a new perspective on learning and innovation", Administrative Science Quarterly, vol. 35, n 1, p. 128-152.

Cooke P. (2001). "From technopôles to Regional Innovation Systems: The Evolution of Localised Technology Development Policy", Canadian Journal of Regional Science, vol. $24, \mathrm{n}^{\circ} 1$, p. 21-40.

Ditter J.G. et Bobulescu R. (2010). «Les systèmes productifs locaux dans les industries du bois : trois études de cas », Revue d'Économie Régionale et Urbaine, 2, mai, p. 269-292.

Drejer I. (2004). "Identifying innovation in surveys of services: A Schumpeterian perspective", Research Policy, vol. 33, n 3, p. 551-562.

Ehlinger S., Perret V. et Chabaud D. (2007). «Quelle gouvernance pour les réseaux territorialisés d'organisation? », Revue française de gestion, vol. 33, n 170, p. 155-171.

Gilly J.P. et Perrat J. (2003). «La dynamique institutionnelle des territoires : entre gouvernance locale et régulation globale », Cahiers du GRES, Université Montesquieux Bordeaux IV, $\mathrm{n}^{\circ} 5$. 
Gilly J.P., Wallet F. (2001). "Forms of proximity, Local Governance and the Dynamics of Local Economic Spaces: The case of Industrial Conversion Processes", International Journal of Urban and Regional Research, vol. 25, n 3, p. 553-570.

Leloup F., Moyart L. et Pecqueur B. (2005). « La gouvernance territoriale comme nouveau mode de coordination territoriale? », Géographie Économie Société, vol. 4, n 7 , p. 321-332.

Lévesque B., Klein J.L., Fontan J.M., Les systèmes industriels localisés : État de la recherche, Montréal, Canada, UQAM.

Longhi C. et Quéré M. (1991). « La technopôle comme système industriel localisé : éléments d'analyse et enseignements empiriques », Économies et Sociétés, 32, p. 21-41.

Loubaresse E. (2008). "Influence des caractéristiques des pilotes de réseaux locaux d'organisations sur leurs rôles », Management International, vol. 13, n 1, p. 86-95.

Loubaresse E. (2011). « Profil généraliste ou profil technique, quand les compétences des pilotes opérationnels influencent le pilotage des Systèmes Productifs Locaux », Revue Management \& Avenir, vol. 10, $\mathrm{n}^{\circ}$ 50, p. 207-225.

Mairesse J. et Mohnen P. (2010). "Using innovations surveys for econometric analysis". NBER WP series 15857 (http://www.nber.org/papers/w15857)

Mendez A. et Bardet M. (2009). «Quelle gouvernance pour les pôles de compétitivité constitués de PME? », Revue française de gestion, vol. 35, n 190, p. 123-142.

Pecqueur B. et Zimmerman J.B. (2004). Économies de Proximités, Paris, Hermès.

Provan K.G. et Kenis P. (2008). "Modes of Network Governance: Structure, Management, and Effectiveness", Journal of Public Administration Research and Theory, vol. 18, $\mathrm{n}^{\circ} 2$, p. 229-252.

Rallet A.et Torre A. (2007). «Faut-il être proches pour innover ensemble? », Quelles proximités pour innover?, Rallet A. et Torre A. (éds), Paris, France, L'Harmattan.

Romijn H. et Albaladejo M. (2002). "Determinants of innovation capability in small electronics and software firms in southeast England", Research Policy, vol. 31, $\mathrm{n}^{\circ} 7$, p. 1053-1067.

Spithoven A., Clarysse B. et Knockaert M. (2011). "Building absorptive capacity to organise inbound open innovation in traditional industries", Technovation, vol. 31, n 1, p. 10-21.

Torre A. (2006). «Clusters et systèmes locaux d'innovation: retour critique sur les hypothèses naturalistes de transmission des connaissances à l'aide des catégories de l'économie de la proximité », Région et Développement, vol. 24, p. 15-43.

Wold H. (1985). "Partial least squares", Encyclopedia of Statistical Sciences, Kots S. et Johnson N.L. (Eds), New York, John Wiley, p. 581-591. 